\title{
GRILLETES DORADOS
}

\section{GOLDEN FETTERS}

Carlos Díaz Gómez: Universidad de Alcalá de Henares. Madrid (España) carlos_diazgomez@yahoo.com

\section{CURRÍCULUM VITAE}

Doctor en Filosofía por la Universidad Complutense de Madrid (España) con Premio Extraordinario, Licenciado en Derecho por la Universidad Complutense de Madrid (España) con Premio Extraordinario, Licenciado en Altos Estudios Constitucionales con Premio Extraordinario y Profesor de la Universidad Complutense de Madrid (España). Autor de numerosos libros y artículos en revistas especializadas y universitarias.

\section{RESUMEN}

El patrón oro fue el mecanismo que transmitió el impacto desestabilizador desde los Estados Unidos al resto del mundo, y el elemento clave para comprender la Gran Depresión de los años treinta. El sistema monetario que ordenaba el comercio internacional antes de la Primera Guerra Mundial era un sistema multilateral de pagos basado también en el oro y con tipos de cambio fijos que parecía tener todo ventajas y pocas limitaciones. Comenzaba así una nueva época. Durante más de un 
cuarto de siglo, con anterioridad a la Primera Guerra Mundial, el patrón oro fue el marco de referencia para las relaciones monetarias nacionales e internacionales.

\title{
PALABRAS CLAVE
}

Oro - Gran Depresión - Sistema monetario - Comercio

\begin{abstract}
The gold standard was the mechanism that transmitted the destabilizing impact from the U.S. to the rest of the world, and the key to understanding the Great Depression of the thirties. The monetary system ordering international trade before the First World War was a multilateral payments system based on gold and also with fixed exchange rates seemed to have all advantages and few limitations. Thus began a new era. For over a quarter century before the First World War, the gold standard was the framework for national and international monetary relations.
\end{abstract}

\section{KEY WORDS}

Gold - Great Depression - Monetary system - Trade

\section{ÍNDICE}

1. Introducción

2. El funcionamiento del patrón oro

3. Las causas de la Gran Depresión

4. El final del patrón oro y el final de la Depresión

5. Conclusión 
6. Notas

TEXTO:

Una recreación en castellano de la casi literalidad de las ideas del Dr. Eichengreen en su libro Golden Fetters

\section{Introducción}

I am ready to admit that gold is a commodity in such general demand that it may always command a market, that it can always buy all other commodities; whereas, other commodities cannot always buy gold. The markets of the world are open to it as merchandise at less sacrifice upon an emergency, than would attend an export of any other article, which might in quantity or kind be beyond the usual demand in the country to which it is sent. So far there can be, I presume, no difference of opinion.

But there will be found to be no inconsiderable difference, if we distinguish as we ought to do, for the purpose whether of theory or practice, between gold considered as merchandise, i.e. as capital, and gold considered as currency circulating in the shape of coin among the public.

...there must be a very considerable amount of the precious metals applicable and applied as the most convenient mode of adjustment of international balances, being a commodity more generally in demand, and less liable to fluctuations in market value than any other.

An Inquiry into the Currency Principle 
Thomas Tooke, London, 1844

La tesis inicial y original o novedosa que defiende el autor en este libro es que el patrón oro de los años veinte es un elemento clave para comprender la Gran Depresión de los años treinta, pues contribuyó a que esta se desatase, al incrementar la fragilidad del sistema financiero internacional. El patrón oro fue el mecanismo que transmitió el impacto desestabilizador desde los Estados Unidos al resto del mundo. Magnificó aquella primera perturbación. Fue el principal obstáculo para la acción correctora; la restricción insalvable o la camisa de fuerza que impidió a los responsables de la política económica de la época evitar las quiebras bancarias y contener la expansión del pánico financiero. La vuelta a la normalidad -la recuperación- sólo fue posible, y por estas mismas razones, cuando el patrón oro fue abandonado.

Como sabemos y hemos estudiado, el sistema monetario que ordenaba el comercio internacional antes de la Primera Guerra Mundial era un sistema multilateral de pagos basado también en el oro y con tipos de cambio fijos que parecía tener todo ventajas y pocas limitaciones. Las razones de estas apreciaciones descansaban naturalmente en el hecho de que el sistema era nuevo en una época nueva, entusiasta e innovadora, que confiaba casi absolutamente en la capacidad de la ciencia y de la técnica para sortear y traspasar las barreras más infranqueables y las dificultades más eternas. Nuevo era el hecho de que los distintos y pocos países que protagonizaban el grueso del comercio internacional en aquel momento se hubiesen puesto de acuerdo para liquidar sus saldos respectivos de forma ordenada, sin invadirse ni liquidarse mutuamente, o unilateralmente, como había venido sucediendo algunas veces en el pasado y como habría de suceder de nuevo en un futuro no muy lejano. El sistema, con sus "puntos oro" y el detalle de su funcionamiento, permitía reducir al máximo los movimientos de la más preciada de todas las mercancías, y dotaba de estabilidad cambiaria, de estabilidad de balanza de pagos y de estabilidad del nivel de precios 
las relaciones comerciales, contribuyendo de este modo a su consolidación y a su desarrollo, gracias a lo cual tuvo lugar un impresionante crecimiento de las economías industriales. De ahí el espejismo de la perfección casi absoluta de aquel sistema. Aún no había sido ensayado suficientemente -no era lo bastante viejo- y se ignoraban sus limitaciones. Se apoyaba en la cooperación internacional y en la credibilidad del compromiso oficial respecto de la convertibilidad oro de las monedas de los países miembros. La credibilidad inducía el capital financiero a moverse en direcciones y sentidos estabilizadores, reforzando la estabilidad económica. Y la cooperación ponía de manifiesto que el sostenimiento del patrón oro en tiempos de crisis superaba la capacidad y los recursos con los que podía contar cualquier país aisladamente. Esta cooperación podía garantizarse -convirtiéndose así en compromiso- en la medida en que los participantes eran pocos y el poder político homogéneo: los partidos obreros no habían llegado aún a los parlamentos del mundo; los costes de negociación eran mínimos. Las cosas ya no serían así después de la guerra. Cuando comenzó a desconfiarse de la convertibilidad, la cooperación internacional se hizo más capital e imprescindible que nunca. Su menoscabo habría de traducirse automáticamente en crisis económicas.

Comenzaba una nueva época. El desacuerdo sobre la distribución de la renta, sobre el papel y el tamaño del estado; y, en el orden internacional, las disputas acerca de las deudas y las reparaciones de guerra hacían cada vez más difícil la cooperación. Economía y política se dieron cita para poner en cuestión, retar y finalmente comprometer la independencia de los banqueros centrales, los guardianes tradicionales del sistema del patrón oro. Los desacuerdos teóricos o doctrinales llevaron a los países a diagnosticar sus males económicos de manera dispar, haciendo de este modo imposibles sus esfuerzos de cooperación para administrar un remedio común. Puesto que estamos hablando de cambios económicos trascendentales que estaban acentuando la fragilidad de las instituciones financieras nacionales e internacionales, este orden de cosas significaba un billete seguro hacia el 
desastre. Los desórdenes monetarios, como siempre sucede, tenían su reflejo en la esfera financiera. Todavía hoy, como entonces y como mañana, se discute, se reflexiona y se trata de averiguar cuáles fueron los mecanismos de transmisión de aquellas alteraciones que acabaron dando lugar a la Gran Depresión, «la gran catástrofe económica de los tiempos modernos», en palabras del autor. Este es el tema esencial de este libro. Eichengreen se propone articular todos los elementos a los que me acabo de referir en una narración y en una descripción coherente de la política económica y del comportamiento de la economía en el período de entreguerras. Su objetivo es evidenciar cómo las políticas que se siguieron, junto con los desequilibrios económicos generados por la Primera Guerra Mundial, dieron lugar a la catástrofe que fue la Gran Depresión. Su tesis es que el patrón oro determinó esencialmente las políticas económicas llevadas a cabo, y que tuvo mucha responsabilidad en la conformación del inestable ambiente económico sobre el que aquellas actuaron.

Porque aquella catástrofe -argumenta- fue un fenómeno global. En contra de la idea expresada en mucha de la literatura, que se centra en los EEUU, la Gran Depresión fue tan severa precisamente porque hubo tantos países que se vieron afectados simultáneamente. Ninguna economía nacional fue inmune. Todas sufrieron dificultades financieras y muchas experimentaron debilitantes crisis financieras. Es por tanto lógico buscar la llave que abre la caja del misterio de la Depresión en las instituciones que conectaban entre sí los mercados financieros de los distintos países.

Y aquí es donde el patrón oro entra en escena. Durante más de un cuarto de siglo con anterioridad a la Primera Guerra Mundial, el patrón oro había sido el marco de referencia para las relaciones monetarias nacionales e internacionales. Las diferentes monedas de los distintos países eran convertibles en oro a voluntad del público y estaban unidas internacionalmente mediante tipos de cambio fijos. Los envíos o embarques de cargamentos de oro eran el último recurso para resolver problemas o 
realizar ajustes de balanza de pagos. Aquel patrón oro había sido un mecanismo muy eficiente y eficaz para la ordenación de los asuntos financieros. Ninguna crisis global comparable con la que comenzó en 1929 había dado al traste con el funcionamiento de los mercados financieros. Ningún revés económico comparable con el que tuvo lugar en los años treinta había deprimido de tal modo la producción y el empleo. No quiere esto decir que no se hubiesen conocido recesiones y pánicos financieros antes de la primera gran guerra. Únicamente, que ninguna tuvo el carácter global ni la severidad de la que comenzó en 1929.

Los elementos centrales de aquel sistema saltaron por los aires con el estallido de la Primera Guerra Mundial. Fue necesaria más de una década para completar su reconstrucción. Pronto se hizo patente que el patrón oro reconstruido era menos resistente, boyante y prometedor que su predecesor. No fue necesario ir más allá de 1929. El nuevo sistema monetario internacional comenzó a crujir. La rápida deflación -caída de los precios- obligó a los países productores de bienes primarios a suspender la convertibilidad oro y a depreciar sus monedas, esto es, a hacer que perdiesen valor con respecto a las demás monedas del sistema. Los problemas de pagos se extendieron a continuación al mundo industrializado. Durante el verano de 1931 Austria y Alemania conocieron pánicos bancarios y decidieron implementar controles de cambios, suspendiendo la convertibilidad de sus monedas. Gran Bretaña, junto con los Estados Unidos y Francia uno de los países del corazón del sistema monetario internacional, sería el siguiente en atravesar una crisis, abandonando el patrón oro en el otoño de 1931. Aproximadamente dos docenas de países decidieron seguir su ejemplo. Los EEUU lo hicieron en 1933; Francia se mantuvo dentro hasta el amargo final, que le llegó en 1936.

Habitualmente se culpa al colapso del sistema monetario internacional de desatar una crisis que transformó una modesta recesión económica en un hundimiento sin precedentes. En tanto se mantuvo el patrón oro -se argumenta- la recesión posterior a 1929 no supuso más que otra contracción cíclica. Pero la suspensión del patrón oro 
destruyó la confianza en la estabilidad financiera, dando lugar a huidas de capital que minaron la solvencia de las instituciones financieras. Las crisis financieras se trasladaban de país a país, haciendo caer a su paso la actividad económica. El abandono del patrón oro intensificó más la crisis. Tras haber suspendido la convertibilidad, los responsables políticos comenzaron a manipular sus monedas, embarcándose en devaluaciones competitivas que tenían por finalidad tratar de salir de la crisis unilateralmente, trasladando el coste del ajuste a los demás países, puesto que una moneda depreciada con respecto a las otras monedas de los países con los que uno determinado tiene relaciones comerciales hace, en principio NOTA1, para aquellos los productos de este más competitivos y dignos de su atención importadora, remunerando con el pago de cada producto importado factores productivos propios de la economía real de ese país, esto es, capital de toda índole y trabajo -hasta donde pueden separarse los factores de producción unos de otros-, estimulando así la actividad real y la recuperación económica del país que ha devaluado, mas no de los demás (salvo que los bienes importados por estos últimos se utilicen para incrementar su propia producción en sectores en los que efectivamente tengan ventaja relativa con respecto a todos o alguno o algunos de los demás, es decir, sean capaces de producir de modo más eficiente, esto es, lo mismo con menor gasto o esfuerzo, o algo nuevo o diferente que los demás quieran o necesiten, en cuyo caso hablaríamos de eficacia superior, un tipo distinto y como más esencial si se quiere de crecimiento de la renta), pues la recuperación propia se consigue aquí a costa de desviar recursos ajenos para remunerar factores propios ¿cómo si no, con una central nuclear?-. Es por esto que se denomina a este tipo de depreciación NOTA2 como de "empobrecer a tu vecino" (beggar-thy-neighbor). Pues bien, en aquella coyuntura, estas devaluaciones, al ser practicadas por todos los países a la vez, no contribuyeron prácticamente nada a estimular la actividad económica en casa al tiempo que sólo conseguían empeorar la Depresión en el exterior. El mundo de las finanzas se hallaba atomizado, dividido en pequeñas áreas 
monetarias competidoras, desbaratando el comercio internacional, desanimando la inversión extranjera, y dificultando en general la recuperación.

De modo que el patrón oro se presenta convencionalmente como sinónimo de estabilidad financiera. Su ruina en 1929 se hace depender de la crisis financiera global y de la depresión mundial. Una idea esencial de este libro es que lo cierto es exactamente lo contrario. Lejos de ser sinónimo de estabilidad, fue precisamente el patrón oro la principal amenaza para la estabilidad financiera y para la prosperidad económica del periodo de entreguerras. Para entender por qué -continúa explicándonos el autor- hemos de apreciar en primer lugar las razones por las cuales el patrón oro restablecido funcionó tan míseramente cuando su antecesor de antes de la guerra había funcionado tan bien. A continuación, debemos identificar las conexiones existentes entre el patrón oro y la Gran Depresión. Por último, para remachar el argumento, nos queda demostrar que el abandono del patrón oro en los años treinta sentó las bases previas necesarias para hacer posible la superación de la Depresión. Estas son las tres tareas de las que este libro se ocupa.

\section{El funcionamiento del patrón oro}

Existe amplio consenso sobre las razones del contraste entre la estabilidad del patrón oro clásico y la inestabilidad de su réplica de entreguerras. La explicación dominante está expresada con la mayor claridad en el trabajo de Charles Kindleberger, quien sostiene que la estabilidad del patrón oro prebélico fue consecuencia de la gestión efectiva de su miembro más importante -Gran Bretaña- y de su agente -el Banco de Inglaterra-. Se considera que el mercado de capitales británico incrementaba sus préstamos al exterior cuando la actividad económica se apagaba, suavizando más que agravando el ciclo internacional de los negocios. Y que el Banco de Inglaterra estabilizaba el sistema actuando como prestamista internacional de última instancia. Kindleberger contrasta la situación prebélica con el periodo de entreguerras, cuando 
el Reino Unido era demasiado débil para estabilizar el sistema y los Estados Unidos no estaban preparados para hacerlo. En una aplicación de lo que ha venido en llamarse la teoría de la estabilidad hegemónica -término acuñado por Keohane en 1980-, Kindleberger concluye que la necesaria influencia estabilizadora sólo era provista convenientemente cuando podía contarse con un poder económico dominante -o hegemónico- en condiciones y con capacidad para tal misión.

Un precursor importante de Kindleberger en este sentido es Brown (1940), quien también llamó la atención sobre las diferencias existentes entre el centro y la periferia del sistema, argumentando que lo inadecuado del patrón oro de entreguerras había de atribuirse a la influencia desestabilizadora de los países del centro.

El capítulo segundo de este libro pone en cuestión este argumento, apuntando que la ausencia de un poder hegemónico no es un rasgo exclusivo del periodo de entreguerras; como tampoco hubo antes de la Primera Guerra Mundial un país que se ocupase en solitario de los asuntos monetarios internacionales. Puede que Londres fuese el centro financiero internacional más importante, mas contaba con rivales de altura, en especial París y Berlín. El patrón oro anterior a la guerra era un sistema multipolar y descentralizado. El hecho de que funcionase bien no puede atribuirse a la intervención estabilizadora de un poder hegemónico.

La estabilidad del sistema anterior a la guerra no era -como ya hemos indicado- sino el resultado de dos factores muy distintos: la credibilidad y la cooperación.

La credibilidad -continúa explicándonos el autor- es la confianza depositada por el público en el compromiso de un gobierno hacia una política determinada. La credibilidad del patrón oro procedía de la prioridad que los gobiernos atribuían al mantenimiento del equilibrio de la balanza de pagos. En cuanto a los principales países del sistema -Reino Unido, Francia y Alemania-, no había demasiadas dudas 
de que las autoridades tomarían cuantas medidas fuesen precisas en defensa de las reservas áureas de sus bancos centrales y del mantenimiento de la convertibilidad oro de sus monedas. Si uno de esos bancos centrales perdía reservas de oro con el consiguiente debilitamiento de su tipo de cambio, acudían fondos desde el exterior anticipándose a las ganancias de capital que los inversores en activos nacionales habrían de realizar tan pronto como las autoridades adoptasen las medidas necesarias para contener la pérdida de sus reservas fortaleciendo consecuentemente su tipo de cambio. Puesto que nadie ponía en duda el compromiso de aquellos gobiernos con la paridad existente, el capital no hacía sino fluir hacia el interior rápidamente y en volumen considerable. El tipo de cambio se fortalecía así por sí solo, y los flujos estabilizadores de capital hacían mínima la necesidad de una intervención por parte de las autoridades. La misma credibilidad del compromiso oficial con respecto al oro hacía que este compromiso fuese rara vez puesto a prueba. En el capítulo 2 se argumenta con detalle que la credibilidad del patrón oro fue considerablemente mayor en el centro que en la periferia y después de 1890 más que con anterioridad a esa fecha.

¿Qué hacía que el compromiso con el oro fuese creíble? En parte, no se percibía con claridad que las políticas precisas para la consecución del equilibrio exterior eran incompatibles con la prosperidad en el interior. Las autoridades apenas acertaban a comprender que la defensa del patrón oro y la reducción del desempleo podrían muy bien ser objetivos difíciles -cuando no imposibles- de casar. El problema del desempleo sólo se presentó como un problema social y económico serio y digno de atención hacia el final de la centuria. Hasta entonces, tanto en la Inglaterra victoriana como en prácticamente todos los países se consideraba que no existía desempleo involuntario, lo cual denota una falta de comprensión de cómo las fluctuaciones agregadas -a las que los contemporáneos se referían como ciclo de los negociosinfluían sobre el nivel de empleo. No se trata de que los observadores de la época no fuesen conscientes de la existencia de desempleados, sino sólo de que no eran 
capaces de relacionar el aumento y la disminución del desempleo con las fluctuaciones macroeconómicas.

Incluso los observadores que sí eran capaces de establecer tal conexión, rara vez relacionaban las fluctuaciones agregadas con los tipos de interés o con las condiciones monetarias. Su apreciación de cómo la política del banco central podía afectar la economía era limitada. No existía una teoría bien articulada capaz de explicar cómo la oferta de dinero y el crédito podían manipularse para estabilizar la producción o reducir el desempleo, al modo de las teorías desarrolladas por Keynes y por otros autores después de la Primera Guerra Mundial. Quienes se dedicaron a estudiar estas variaciones en el crédito y en el dinero, como por ejemplo Ralph Hawtrey en 1913, acabaron por concluir que no hacían sino intensificar de modo perverso el ciclo de los negocios. En lugar de abogar por una gestión activa de las condiciones monetarias para estabilizar la economía, la mayoría de los observadores aconsejaban una actitud pasiva y por tanto predecible.

Las clases trabajadoras, desprovistas de poder político suficiente, no estaban en condiciones de poner en cuestión este estado de cosas. Así que las presiones políticas domésticas no conseguían minar la credibilidad del compromiso con el oro.

Esta cuestión no debe en cualquier caso exagerarse. Antes de que terminase la primera década del siglo veinte, el desempleo se había convertido en un problema social de primer orden.

La generalización de los sindicatos y la extensión de las franquicias habían aumentado la influencia política de quienes se encontraban en una situación más vulnerable cuando se destruía empleo. Cada vez se estaba más de acuerdo en que los altos tipos de interés desanimaban la inversión y deprimían el comercio. Los 
banqueros centrales no eran insensibles a estas consideraciones. Sin embargo, cuando se veían obligados a elegir entre objetivos externos e internos, lo tenían claro.

Los responsables de la política económica tampoco creían que los déficit presupuestarios o el aumento del gasto público pudiesen utilizarse para estabilizar la economía. Puesto que los gobernantes se ceñían a la regla del presupuesto equilibrado, eran las variaciones en los ingresos las que determinaban los cambios en el nivel de gasto público. Rara vez se encontraban, por lo tanto, los países en un brete enfrentados con la necesidad de eliminar grandes déficit presupuestarios con el fin de poner freno a las salidas de oro. Había normas claramente establecidas en cuanto a la distribución de la carga fiscal. En lo que se refiere a los ingresos, los gobiernos centrales contaban en primer lugar con los impuestos a la importación (derechos de aduana); la recaudación de los impuestos sobre la renta y sobre la actividad en el interior era aún muy costosa. Los individuos a quienes se les exigía el pago de los derechos de aduana -habitualmente adquirentes de productos alimenticios de importación y otros bienes de consumo- solían ser perceptores de salarios con relativamente poca voz política. Cuando las necesidades de ingresos cambiaban, los impuestos a la importación podían ajustarse convenientemente. La necesidad de eliminar un déficit presupuestario no desataba automáticamente un acalorado debate sobre temas impositivos. Los gobiernos podían comprometerse de manera creíble a dirigir tanto sus instrumentos fiscales como monetarios hacia objetivos de balanza de pagos.

Así que una constelación muy particular de poderes políticos, reforzada por influyentes instituciones políticas, y una visión concreta del funcionamiento de la economía constituían los fundamentos del sistema del patrón oro clásico. Esta combinación de factores -instituciones políticas e influencia, por un lado, y marco conceptual imperante, por otro- constituían la base de la credibilidad del sistema. 
Sin embargo, la credibilidad del patrón oro clásico descansaba en última instancia en la cooperación internacional. Cuando tanto la especulación estabilizadora como la intervención del gobierno se mostraban incapaces de neutralizar una perturbación, la estabilización del sistema se lograba gracias a la cooperación entre gobiernos y bancos centrales. El autor refiere aquí cómo Kenen (1990) establece una útil distinción entre cooperación económica internacional y coordinación internacional de la política económica. La cooperación puede adoptar formas diversas, tales como, por ejemplo, la asistencia financiera directa a países extranjeros. La coordinación internacional es una forma de cooperación en la cual varios gobiernos acuerdan modificar sus políticas de modos que serían indeseables si se llevasen a cabo de forma unilateral, pero que mejoran sus posiciones relativas cuando se acometen por todos ellos de forma conjunta.

Cuando utilizo el término "cooperación" -prosigue nuestro autor- he tratado de poner el acento en las respuestas de colaboración distintas de los ajustes mutuamente beneficiosos referidos a políticas nacionales que son el centro de atención de la literatura sobre coordinación de políticas; estas otras respuestas de colaboración a las que me refiero incluyen la asistencia financiera directa y los cambios unilaterales en la política de un estado diseñados para aliviar las presiones económicas exteriores, aunque habrá también ocasiones en las cuales haga especial hincapié en los beneficios de la coordinación de políticas definida de modo restringido. Mi noción de cooperación difiere de la de "prestamista internacional de última instancia" de Kindleberger en mi insistencia en la naturaleza multilateral de las políticas requeridas, así como en resaltar la importancia de las otras respuestas distintas de la simple asistencia financiera internacional. Kenen distingue además la colaboración que permite a los funcionarios lograr ciertos objetivos económicos (mejorar el equilibrio entre inflación y desempleo, por ejemplo) de la que tiene por objeto preservar un régimen económico determinado (defender con éxito el patrón oro, pongamos por caso). Buena parte de la cooperación en la que yo me detengo era del 
tipo de preservación de un régimen, aunque, como tendremos ocasión de ver, tenían sus repercusiones, muy considerables, en la capacidad de los gobiernos para alcanzar objetivos económicos inmediatos.

Como resumen de este epígrafe, podemos decir -termina Eichengreen- que el buen funcionamiento del patrón oro clásico descansaba en la credibilidad y en la cooperación. El alcance de ambas se vio mermado repentinamente con la llegada de la Primera Guerra Mundial. El resultado inevitable, la inestabilidad del patrón oro del periodo de entreguerras.

\section{Las causas de la Gran Depresión}

El vínculo que une el patrón oro con la Gran Depresión tiene su origen en los cambios operados en el modelo de liquidación de saldos de balanza de pagos legado por la Primera Guerra Mundial. La guerra fortaleció enormemente la posición de balanza de pagos de los EEUU y debilitó las de otras naciones. A mediados de los años veinte, las cuentas exteriores de ciertos países se mantuvieron ligeramente equilibradas gracias a las salidas de capital a largo plazo procedente de los EEUU. Pero si el crédito americano se interrumpía, la debilidad subyacente tras las posiciones exteriores de esos otros países afloraría de súbito. A medida que fuesen perdiendo oro y divisas, la convertibilidad oro de sus monedas se vería comprometida. Sus bancos centrales se verían obligados a restringir el crédito interior, sus autoridades fiscales a reducir el gasto público, aunque al hacerlo se arriesgasen a hundir a sus economías en la recesión.

Eso fue lo que sucedió cuando en el verano de 1928 el crédito estadounidense se redujo como consecuencia de una política monetaria cada vez más restrictiva por parte de la Reserva Federal, el banco central del sistema financiero americano. Quiso la fortuna que la contracción en los Estados Unidos coincidiese con la existencia de 
un flujo masivo de oro hacia Francia, donde la política monetaria también estaba siendo restrictiva, aunque por otras razones, que son las que siguen, tal y como nos las refiere el autor. Cuando a finales de 1926 la inflación se hubo detenido y el franco estabilizado, tuvo lugar en Francia un tremendo aumento en la demanda de dinero. Pero nuevas restricciones legales aplicables al Banco de Francia le impedían satisfacer esa demanda comprando títulos e incrementando la cantidad de dinero en circulación.

El único modo de poder atender esa demanda adicional de dinero era recurrir a las importaciones de oro. Al final, la oferta monetaria creció rápidamente en Francia en 1928, pero sólo como consecuencia de esas entradas de oro. Es en este sentido en el que la política monetaria fue restrictiva en Francia a finales de los años veinte. En el capítulo 7 del libro se explica con detalle este episodio. Yo no he pasado de hacer sino un vuelo de reconocimiento a grandísima velocidad. Sus treinta páginas requieren una lectura tan reposada y estricta, que no pueden ser objeto más que de continuos ataques, seguidos de largos paseos y largas horas de meditación. Y eso sólo puede pertenecer al mundo del futuro. La sola traducción de este capítulo siete muy bien podría haber sido un tema para el trabajo que usted nos ha encomendado, ¿verdad? NOTA3

Nos encontrábamos en el momento en que se había reducido el crédito estadounidense a otros países en el verano de 1928 como consecuencia de una política monetaria restrictiva por parte de la Reserva Federal que tenía por objeto frenar los excesos especulativos que se estaban produciendo desde hacía años en la bolsa de Nueva York. Y habíamos anotado también la entrada de grandes cantidades de oro en Francia. De modo que oro y capital financiero estaban siendo absorbidos por los Estados Unidos y por Francia a expensas del resto del mundo. Añadidos a su ya de por sí débil situación de balanza de pagos, estos acontecimientos provocaron una fuertemente magnificada contracción monetaria en el exterior. Precipitaron, 
además, un endurecimiento de las políticas fiscales en zonas de Europa y en la mayor parte de Iberoamérica. Este cambio de política en todo el mundo -y no el simple relativamente pequeño cambio sin importancia que se produjo en los Estados Unidos- dio lugar al impulso contractivo que sentó las bases del colapso de 1929. Un pequeño cambio de orientación en la política estadounidense tuvo efectos tan demoledores a consecuencia de la reacción en el exterior a que dio lugar, a través de su interacción con los desequilibrios presentes en la situación mundial de las balanzas de pagos y con las restricciones impuestas por el patrón oro.

Esta explicación del comienzo de la Depresión, que destaca los cambios simultáneos que se produjeron en las políticas económicas del exterior y de los Estados Unidos, conectados por el patrón oro, así como el impacto conjunto de las políticas estadounidenses y no estadounidenses sobre el nivel de actividad, no ha aparecido con anterioridad en la literatura -culmina nuestro autor-. Sus elementos son bien conocidos, pero nadie nunca los había expuesto conjuntamente para dar cuenta cabal de las causas del hundimiento económico acaecido en 1929.

\section{El final del patrón oro y el final de la Depresión}

Si el patrón oro contribuyó a la severidad de la crisis, ¿libró su colapso al mundo de la atenazadora Depresión? De acuerdo con la sabiduría convencional, la depreciación monetaria que pudo llevarse a cabo tras el abandono del patrón oro no logró mejorar las condiciones económicas en los países que suspendieron su convertibilidad, y agravó la Depresión en aquellos que permanecieron vinculados al sistema.

Nada más lejos de la realidad. La depreciación fue la clave del crecimiento económico. Allí donde se intentó, casi siempre contribuyó a estimular la recuperación económica. En los países que se desvincularon del oro los precios se estabilizaron. La producción, el empleo, la inversión y las exportaciones crecieron 
más rápidamente que en aquellos países que decidieron mantener sus monedas vinculadas a sus paridades oro.

La ventaja de la depreciación consistía en que permitía recuperar la libertad de las políticas monetarias y fiscales. Ya no era preciso limitar el crédito interior para defender la convertibilidad. Ya no era preciso reducir el gasto público en países donde ya se hallaba de por sí muy reducido. «Hay pocos hombres ingleses a quienes no les llene de dicha la idea de romper nuestros grilletes dorados», tal y como Keynes lo expresó en septiembre de 1931 cuando su país se vio obligado a devaluar. No era sólo el patrón oro como conjunto de instituciones lo que dificultaba la recuperación económica, sino también su espíritu. Aunque para poder adoptar medidas de reflación era preciso abandonar la convertibilidad, no bastaba con eso. Una crisis financiera podía obligar a un país a abandonar la convertibilidad, pero eso no significaba que tuviese que abandonar la ortodoxia financiera. La recuperación sólo siguió cuando también se hubo renunciado a los principios de la ortodoxia financiera.

\section{Conclusión}

By the definition of the balance of payments, the balance of current account, the balance of capital account, and gold movements must sum zero. This implied that swings in foreign lending could take place in the absence of large-scale gold movement only if they were followed without delay by corresponding swings in the current account and specifically in the balance of commodity trade.

Barry Eichengreen, California, 1992

Según la definición de balanza de pagos, la balanza por cuenta corriente, la balanza por cuenta de capital y los movimientos de oro han de sumar cero. Lo cual implicaba 
que las variaciones en los préstamos procedentes del exterior sólo podían tener lugar, en ausencia de movimientos de oro a gran escala, si eran seguidas sin demora por las correspondientes variaciones en la balanza por cuenta corriente, y en concreto, en la balanza de mercancías.

\section{Notas}

1. Es imprescindible que no cambien los precios relativos hasta el punto de dejar sin efecto la devaluación ni se modifique, reduciéndose, el volumen de importaciones y exportaciones de bienes y servicios, ni la cuenta de capital: ¿demasiadas cosas como para extraer de un principio teórico una proposición empírica significativa? Véase la denominada condición Marshall-Lerner, y téngase muy presente que hay cinco teorías para explicar la determinación de los tipos de cambio. (Véase exchange rates en The New Palgrave Dictionary of Money and Finance).

2. Los términos "devaluación" y "depreciación" sólo se distinguen en que éste suele aplicarse en sistemas de tipos de cambio flexibles, mientras que aquél se reserva para los caracterizados por tipos de cambio fijos.

3. La primera versión de este trabajo la realicé en cumplimiento de las exigencias de la asignatura Historia económica internacional de los siglos XIX y XX, a cargo del profesor Martín Aceña, el Milton Friedman español, me digo a mí mismo al leer los títulos de su obra escrita. Sus clases -interesantísimas conferencias de cien minutos los lunes y martes de tres a cinco- realmente contribuyeron grandemente a nuestra realización inagotable de ganancias de capital intelectual. El profesor Torrero siempre preocupado de nuestra formación científica- me recomendó la lectura del 
libro cuando me encontraba en segundo curso y él nos había hablado de la importancia y actualidad del tema del patrón oro y se había brindado a facilitar referencias bibliográficas concretas a cuantos pudieran estar interesados en la cuestión. Casi seis años después y a punto de licenciarme, aún no he sido capaz de leerlo con seriedad, pero noto que mi productividad o asimilación de literatura económica avanza lentamente. 